\title{
Skin Lesion Classification: A CNN Way
}

\author{
Prasad Thakar, Siddhivinayak A Kulkarni
}

\begin{abstract}
Skin lesion growth of unwanted cells on the upper most layer of skin. These lesions may conation cancerous cells which may lead to health issues to the patient and in severe cases may lead to patient's demise. Dermatologists identify type of skin cancer by identifying it in image generated using dermatoscope and procedure known as Dermatoscopy. Previously there have been many studies which show classification of these dermatoscopic images using machine learning and deep learning solutions. Machine learning approaches use image processing techniques for identifying mole in given image and then for classification researchers have used techniques like SVM , random forest etc. With advances in field of deep learning there have been various methods proposed on classification of using CNN which achieves more precision and accuracy. In this paper we are proposing a CNN based approach for image classification with best overall accuracy of $78.08 \%$ and good multiclass AUC for all classes in HAM10000 dataset.

Keywords: Artificial Intelligence, Computer Vision, Deep Learning, Image Classification, Skin lesion classification, Convolutional-Neural-Network.
\end{abstract}

\section{INTRODUCTION}

Skin cancer is one of the fatal diseases, every it causes fatality all around the globe and also many new cases are being discovered every year. Skin cancers are generally start developing on the upper most layer of skin or epidermis. These legions grow in size and shape and can reach up to lymph nodes which might then be spread to distant organs also via transmission which further leads to critical illness and in few cases death of patient. Skin cancers are further divided into two main types melanoma and non-melanoma cancer.

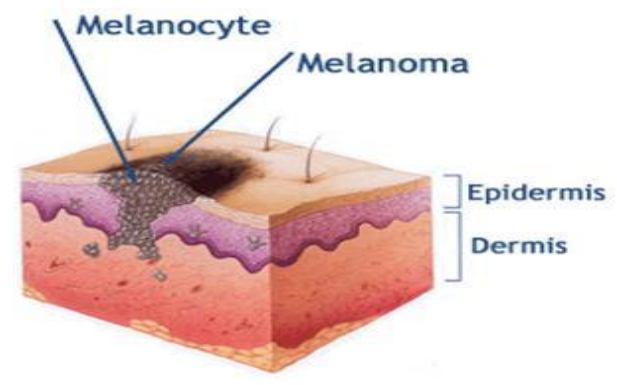

Fig.1 Skin cross section of melanoma[18]

Revised Manuscript Received on May 15, 2020.

* Correspondence Author

Prasad Thakar*, School of Computer Engineering and technology, MIT World Peace University, Pune, Maharashtra, India. Email: tprasad31@yahoo.com

Dr. Siddhivinayak A Kulkarni, School of Computer Engineering and technology, MIT World Peace University, Pune, Maharashtra, India. Email: Siddhivinayak.kulkarni@mitwpu.edu.in

(C) The Authors. Published by Blue Eyes Intelligence Engineering and Sciences Publication (BEIESP). This is an open access article under the CC BY-NC-ND license (http://creativecommons.org/licenses/by-nc-nd/4.0/)
More than 9500 cases of melanoma cancer are discovered every single day. In united states skin cancer causes death of more than 2 people every day. One of every five American at the age of 70 will develop skin cancer[16]. More than a million people in US are suffering from melanoma cancer[17]. During past decade number of newly diagnosed melanoma cases are increased by $47 \%[16]$. Last year more than 7200 deaths were occurred due to melanoma [17] and this year (2020) it is projected to be 6850[16]. Melanoma in children accounts for $1-4 \%$ of overall melanoma cases and $3 \%$ of pediatric cancers [16]. $40 \%$ times there is treatment delay in pediatric melanoma cancer due to mis-diagnosis[16]. Every year Basel cell carcinoma(BCC) cases in US are estimated to have number of 4.3 million[16]. Rate of increase in cases of BCC are increased by $145 \%$ during 2000-2010[17]. Actinic Keratosis is one of most commonly found pre-cancer stage which is said to be affected 58 million Americans[16]. Every month more than 5400 people die due to non-melanoma cancer worldwide[16]. In Asia skin cancer accounts for $2 \%$ to $4 \%$ of all cancers detected in the region[16]. It has been observed that $99 \%$ of melanoma patients' can survive within 5 years span if it is diagnosed in early stages [16]. Five-year survival chances for regional and distant stage melanoma is $64 \%$ and $23 \%$ respectively[17].

In this paper, section 2 discusses about various proven techniques by various researchers. Section 3 describes about dataset used for proposed method. Section 4 explains about our method for classification of skin lesions and section 5 describes about our experimentation on proposed method with comparison with similar techniques from other researchers.

\section{LITERATURE SURVEY}

Various researchers have worked on classification of pigmented skin images using machine intelligent approaches. During their study they have used various image processing techniques with combination of traditional machine learning algorithms like SVM, random forest, Bayesian learning for classification. Some studies also been proposed on artificial neural network-based classification of skin lesions. With advances in fields of deep learning and computer vison, deep networks are used widely for image classification in medical domain studies. Researchers have also worked on CNN,RNN models for image classifications.Suleiman Mustafa et al. [1] has proposed a Support Vector Machine(SVM) based method for classification based on lesion's colour and texture of skin lesion. Soniya et al. [3] worked on feature detection for skin moles using OTSU's thresholding approach. Zahara et al. [4] has worked on SVM based intelligent machine learning model for classification with Gray-Level Co-Occurrence Matrix (GLCM) based technique for extraction features from images. Prachaya et al. [5] stated SVM model for image classification and snake algorithm for lesion segmentation. NCF Codella et al.

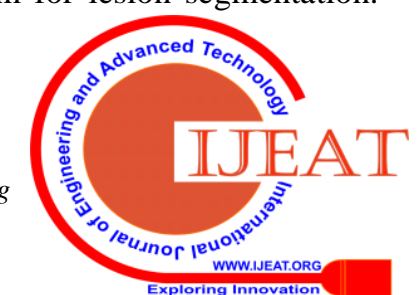




\section{Skin Lesion Classification: A CNN Way}

[5] has proposed on an ensemble learning approach for classification of skin lesions with an accuracy score of 0.77 . Rashika Mishra et al [6] proposed a deep learning solution based on CNN for skin lesion segmentation. Nikhil Cheerla et al.

[7] proposed multi-stage neural network for classification of images. Kiran Pai et al. [8] proposed VGG based deep learning network for classification with an accuracy of $79.56 \%$ with 50 epochs. Tri cong pham [9] worked on method for classification based on balanced random forest approach for classification of images with $74.75 \%$ classification accuracy and HSV based feature extraction on linearly normalized images. D. Moldovan [10] proposed a model with 2 step approach for classification of images in HAM10000 dataset based on DenseNET121 architecture with accuracy of $85 \%$ and $75 \%$ respectively in both steps. . K. E. Purnama et al.[11] worked on model based on MobileNET and InceptionV3 with classification accuracy of $58 \%$ and $72 \%$ respectively. S. Bassi et al. [12] proposed classification of pigmented skin images based on CNN with various pretrained models like VGG16,VGG19,InceptionV3, MobileNET with highest accuracy results via VGG i.e. 82.8\%.

As per the studies done by various researchers classification of pigmented lesions with traditional machine learning techniques combined with image processing can achieve good accuracy but it is limited to small amount of data. Also for multiclass classification class imbalance problem in data can be challenging for better overall classification accuracy. With new advancements in computational deep learning CNN based methods looks as an promising approach for classification of pigmented skin lesions.

\section{DATASET}

Human Against Machine(HAM10000) is the dermatoscopy with collection of 10015 dermatoscpy images created with intention to solve problem of diversification of available data in field of dermatology. These images are of seven different types represented by individual classes. All images are collected by various SMEs considering samples from different age groups, gender and skin tones. This dataset was used as a training dataset for ISIC-2018 challenge hosted by International skin imaging collaboration[22].
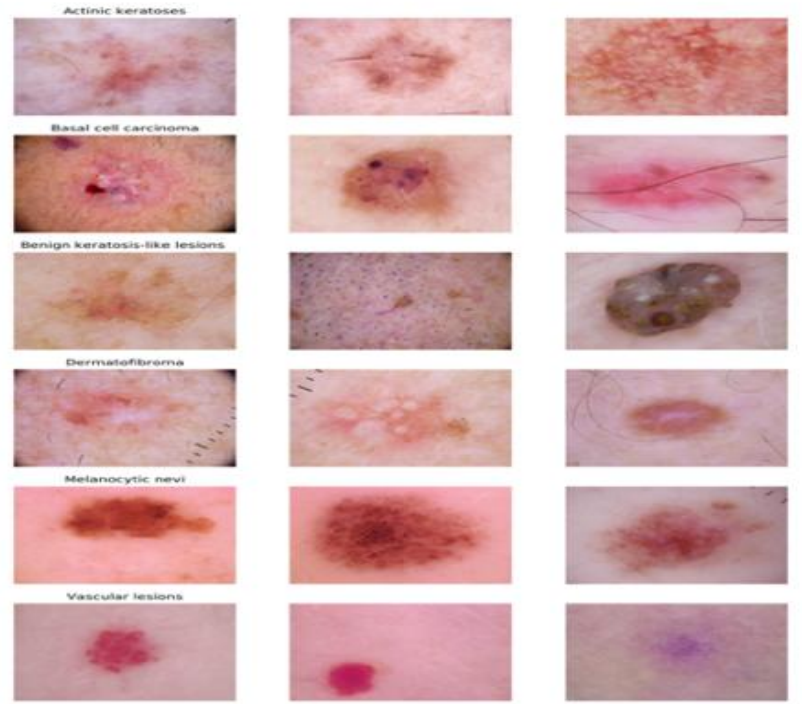

\section{Fig-2: HAM10000 Dataset Samples [21]}

\section{Proposed SysteM}

With advances in the field of deep learning and artificial neural networks, researchers have proven the advantages of these kinds of systems over traditional machine learning methods. In this paper we are proposing a convolutional neural network (CNN) based method classification of pigmented skin images.

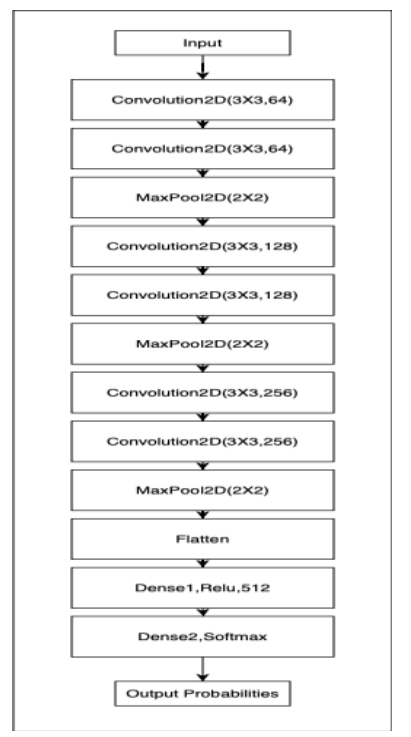

Fig-3 Proposed System Architecture

Above Figure-3 shows the proposed system architecture with various CNN layers. The overall architecture is explained in four different blocks. First three blocks are combination of convolution and pooling layers for feature extraction and fourth one consists of dense layer which will act as classification layer where learnt features will be used classify the image into a particular type or class. First block is part of input a network where 2 convolutional (2D-Convolution) layers with kernel of size 3X3 with feature learning parameter of 64 filters. The first step convolution generally acts as a learning of edges and borders in the image so 64 filters will create significant output for understanding those parts of image. After this the pooling layer with size $2 \mathrm{X} 2$ is added for understanding feature maps generated by convolution layers.

Similarly, block 2 will take the output of first convolved layer as an input, this layer also has kernel size of 3X3 for convolution but now learnable fitters are increased to 128 as we want to learn more complex structures in image than pervious due to reduction of image size done using previous convolution. After convolution layers we have added similar pooling layer for understanding these feature maps. For block three we set convolution of same kernel but now filters have been increased to 256 as this is last layer convolution and we have to most complex features of image and map them for better classification results. After this the we will convert these output feature maps to 1-D tensor for better results. This flattened output is input to first Dense layer which has its own 512 feature maps for learning and activated with Rectified Linear Unit (RELU) function.

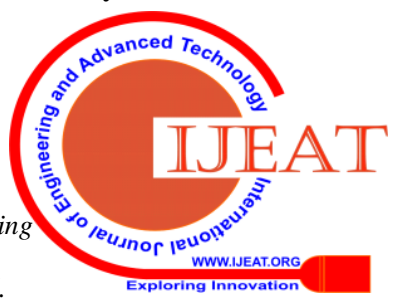


The last layer is SoftMax activation-based dense layer which will give output probability class for given image.

\section{Handing Class Imbalance in Dataset HAM1000}

HAM10000 dataset is collection of skin lesion images of seven different classes. The collection has imbalance in itself for various classes with 6705 images for Melanocytic Nevi (NV or 4) class and 115 images for Dermatofibroma (Df or 3) class. This is tremendous amount of class imbalance and needs to be addressed while training optimizing network for each class efficiently. This can be done using assigning class weights while training the model.

Where,

$$
W_{a}=\frac{N}{N_{c} * N_{a}}
$$

$\mathrm{W}_{\mathrm{a}}$ is class weight for class $\mathrm{a}$.

$\mathrm{N}$ is no of samples.

$\mathrm{N}_{\mathrm{c}}$ is no of unique classes for classification.

$\mathrm{N}_{\mathrm{a}}$ is no of samples in class a.

Table: 1 Class Names and assigned class weights

\begin{tabular}{|l|l|l|l|}
\hline $\begin{array}{l}\text { Categorical } \\
\text { Label }\end{array}$ & Code & Name & $\begin{array}{l}\text { Class } \\
\text { Weight }\end{array}$ \\
\hline 0 & akiec & Actinic Keratosis & 4.2867 \\
\hline 1 & bcc & $\begin{array}{l}\text { Basel Cell } \\
\text { carcinoma }\end{array}$ & 2.7447 \\
\hline 2 & bkl & $\begin{array}{l}\text { Benign } \\
\text { Keratosis-like } \\
\text { lesions }\end{array}$ & 1.308 \\
\hline 3 & df & $\begin{array}{l}\text { Dermatofibroma } \\
\text { Melanocytic Nevi }\end{array}$ & 0.21254 \\
\hline 4 & nv & $\begin{array}{l}\text { Malignant } \\
\text { Melanoma }\end{array}$ & 1.32015 \\
\hline 5 & mel & Vascular lesions & 10.12895 \\
\hline 6 & vasc & \multicolumn{2}{|l}{} \\
\hline
\end{tabular}

\section{EXPERIMENTS}

We have built our proposed model using Keras API in python language with TensorFlow as backend engine.

For these experiments we kept image size of $150 * 100$ $\left(\mathrm{W}^{*} \mathrm{H}\right)$, which has been reduced for each image from original size $(650 * 400)$.

\section{Data Augmentation:}

In order to make model more robust and stable for predictions we have done various data augmentation measures for training of model. This data augmentation for the experiments is done using Keras's Imagedatageneartor module, which applies real-time augmentation on batches of image tensors. This method also expands training size of data by applying various transformations resulting in more skillful models.

- Rotation Range: Images will be rotated within specified image rotation range of degrees for more robust learning.

- Zoom Range: Adding random zoom on image which will focus on region of interest for better segmentation and classification.

- $\quad$ Width and Height Shift: This allows shift of pixels in intended direction while keeping output and input size same.
- Horizontal Flipping: This will create random flipping on images w.r.t. to their horizontal axes which makes more suitable for pigmented skin images compared to vertical flipping of images.

\section{RESULTS}

For above mentioned experiments we trained proposed model with 30 and 50 epochs with optimizers like Adam[19] and Nadam (or Adam+Netstrov's Momentum). Adam is popular stochastic optimizer for neural network which is helpful in reducing the learning rate and adjust the weights of network to reduce losses. We have used starting learning rate of $1 \mathrm{e}^{-3}$ for both optimizers with keeping up beta1=0.9 and beta2 $=0.9999$ both default parameters of ADAM. For annealer (annealing algorithm), we have selected ReduceLROnPlateau which will eventually reduce learning rate of learning of model if there is no change on set parameter with given patience. We will reduce learning rate of algorithm by half if there is no change on validation accuracy with 3 epochs with minimum learning rate of $1 e^{-7}$

$$
\text { New } L R=0.5 * \text { Current } L R
$$

For first iteration of annealer

$$
\text { New } L R=0.5 * 0.0001=0.00005
$$

Table:2 Accuracy \& Loss of Proposed Model

\begin{tabular}{|l|l|l|l|l|l|}
\hline $\begin{array}{l}\text { Model + } \\
\text { Optimizer }\end{array}$ & Epochs & $\begin{array}{l}\text { Val. } \\
\text { Acc } \\
(\%)\end{array}$ & $\begin{array}{l}\text { Val. } \\
\text { Loss }\end{array}$ & $\begin{array}{l}\text { Test } \\
\text { Acc } \\
\mathbf{( \% )}\end{array}$ & $\begin{array}{l}\text { Test } \\
\text { Loss }\end{array}$ \\
\hline $\begin{array}{l}\text { Model + } \\
\text { Adam }\end{array}$ & 30 & 79.3 & 0.588 & 76.73 & 0.6112 \\
\hline $\begin{array}{l}\text { Model + } \\
\text { Adam }\end{array}$ & 50 & 78.55 & 0.5811 & 77.28 & 0.605 \\
\hline $\begin{array}{l}\text { Model + } \\
\text { Nadam }\end{array}$ & 30 & 79 & 0.58 & 78.08 & 0.58 \\
\hline $\begin{array}{l}\text { Model + } \\
\text { Nadam }\end{array}$ & 50 & 78 & 0.56 & 77.28 & 0.59 \\
\hline
\end{tabular}

According to above Table-2 with model and optimizer as NADAM we are getting more classification accuracy with less loss. With this setup model has also achieved great Area under Curve scores for each class greater than $90 \%$.

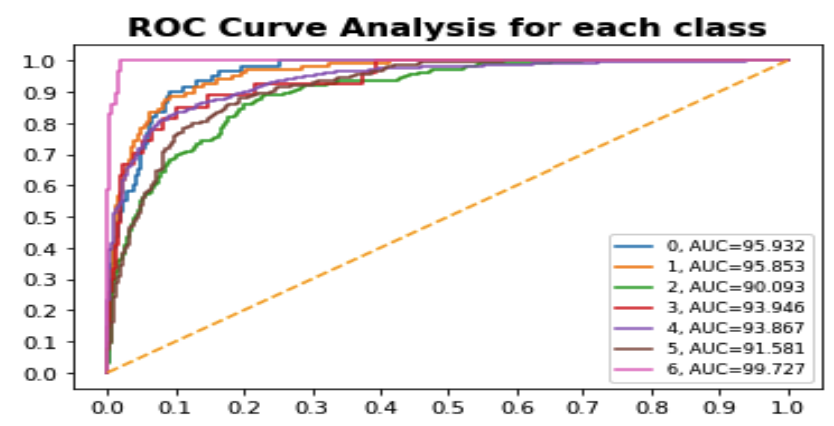

Fig:4 ROC Curve With 30 epochs (NADAM)

Published By:

Blue Eyes Intelligence Engineering \& Sciences Publication 


\section{Skin Lesion Classification: A CNN Way}

Table:3 Classification Report Model with 30 epochs (NADAM)

\begin{tabular}{|l|l|l|l|l|}
\hline $\begin{array}{l}\text { Class } \\
\text { Label }\end{array}$ & precision & recall & f1-score & support \\
\hline 0 & 0.49 & 0.43 & 0.46 & 60 \\
\hline 1 & 0.54 & 0.63 & 0.58 & 97 \\
\hline 2 & 0.49 & 0.63 & 0.55 & 224 \\
\hline 3 & 0.46 & 0.22 & 0.3 & 27 \\
\hline 4 & 0.89 & 0.91 & 0.9 & 1320 \\
\hline 5 & 0.65 & 0.45 & 0.53 & 246 \\
\hline 6 & 0.84 & 0.72 & 0.78 & 29 \\
\hline
\end{tabular}

Comparison with other methods:

Table:4 Comparison with other methods

\begin{tabular}{|l|l|l|}
\hline Method & \multicolumn{2}{|l|}{ Accuracy (\%) } \\
\hline \multirow{2}{*}{ Kiran Pai et al. } & VGG+ 30 Epochs & 78.49 \\
\cline { 2 - 3 } & VGG+ 50 Epochs & 79.56 \\
\hline $\begin{array}{c}\text { Tri Cong Pham } \\
\text { et al }\end{array}$ & Random Forest & 74 \\
\hline $\begin{array}{l}\text { I Ketut Eddy } \\
\text { Purnama et. } \\
\text { al. }\end{array}$ & Mobilenet V1 & 58 \\
\cline { 2 - 3 } & Inception v3 & 78 \\
\hline \multirow{2}{*}{\begin{tabular}{c} 
Bassi et al. \\
\cline { 2 - 3 }
\end{tabular}} & Vgg16 & 82.8 \\
\cline { 2 - 3 } & Vgg19 & 79.4 \\
\cline { 2 - 3 } & Inception V3 & 74.8 \\
\hline \multirow{2}{*}{$\begin{array}{l}\text { Our method } \\
\text { (NADAM) }\end{array}$} & 30 epochs & 76 \\
\cline { 2 - 3 } & 50 epochs & 77.28 \\
\hline
\end{tabular}

\section{CONCLUSION}

In this paper, we had talked deep learning based solution for classification of pigmented skin images. With our approach of deep learning with convolutional neural network(CNN) and experimental setup we have been able to achieve good accuracy score for validation (79\%) and test (78\%) for overall classification of image in seven different classes of HAM10000 dataset. Comparing with some similar techniques our approach has been fairly able to outperform some methods with good accuracy and computation efficiency. The model has been able achieve good AUC score for each class i.e. greater than $90 \%$ individually.

As a future part of work the model can be used to build the application which will classify the given pigmented image to appropriate class which will may a vital role in early diagnosis of disease and giving physicians proper time to plan treatment for the patient. In order to improve the model's accuracy deep learning techniques like RNN,LSTM may contribute to improve further overall accuracy of model.

\section{REFERENCES}

1. Suleiman Mustafa \& Akio Kimura A SVM-based diagnosis of melanoma using only useful image features, 2018 International Workshop on Advanced Image Technology (IWAIT),7-9 Jan. 2018

2. Soniya Mane \& Swati Shinde A Method for Melanoma Skin Cancer Detection Using Dermoscopy Images, 2018 IEEE Fourth International Conference on Computing Communication Control and Automation (ICCUBEA),16-18 Aug. 2018

3. Zahra Waheed, Amna Waheed, Madeeha Zafar \& Farhan Riaz An Efficient Machine Learning Approach for the Detection of Melanoma using Dermoscopic Images,IEEE 2017 International Conference on Communication, Computing and Digital Systems (C-CODE), 8-9 March 2017.

4. Prachya Bumrungkun, Kosin Chamnongthai \& Wisarn Patchoo Detection of skin cancer using SVM and snake model, 2018 IEEE International Workshop on Advanced Image Technology (IWAIT), 7-9 Jan. 2018

5. N. C. F. Codella, Q.-B. Nguyen, S. Pankanti, D. A. Gutman, B. Helba, A. C. Halpern J. R. Smith, Deep learning ensembles for melanoma recognition in dermoscopy images, IBM J. RES. \& DEV. VOL. 61 NO. 4/5 PAPER 5 JULY/SEPTEMBER 2017

6. Rashika Mishra \& Ovidiu Daescu, Deep Learning for Skin Lesion Segmentation, 2017 IEEE International Conference on Bioinformatics and Biomedicine (BIBM)

7. Nikhil Cheerla, Debbie Frazier, Automatic Melanoma Detection Using Multi-Stage Neural Networks, International Journal of Innovative Research in Science,

8. Engineering and Technology Vol. 3, Issue 2, February 2014

9. K. Pai and A. Giridharan, "Convolutional Neural Networks for classifying skin lesions," TENCON 2019 - 2019 IEEE Region 10 Conference (TENCON), Kochi, India, 2019, pp. 1794-1796.

10. T. C. Pham, G. S. Tran, T. P. Nghiem, A. Doucet, C. M. Luong and V. Hoang, "A Comparative Study for Classification of Skin Cancer," 2019 International Conference on System Science and Engineering (ICSSE), Dong Hoi, Vietnam, 2019, pp. 267-272.

11. D. Moldovan, "Transfer Learning Based Method for Two-Step Skin Cancer Images Classification," 2019 E-Health and Bioengineering Conference (EHB), Iasi, Romania, 2019, pp. 1-4.

12. I. K. E. Purnama et al., "Disease Classification based on Dermoscopic Skin Images Using Convolutional Neural Network in Teledermatology System," 2019 International Conference on Computer Engineering Network, and Intelligent Multimedia (CENIM), Surabaya, Indonesia, 2019, pp. 1-5.

13. S. Bassi and A. Gomekar, "Deep Learning Diagnosis of Pigmented Skin Lesions," 2019 10th International Conference on Computing, Communication and Networking Technologies (ICCCNT), Kanpur, India, 2019, pp. 1-6.

14. Skin Cancer: Facts, Statistics, and You. [Online]. Available: https://www.healthline.com/health/skin-cancer/facts-and-stats,

15. Last Accessed:25 th Apr 2020

16. Overview of Non-Melanoma Skin Cancer. [Online] Available: https://www.ahns.info/patient-information/understanding-skin-cancer/ overview/ Last Accessed:25 th Apr 2020

17. Cancer Stats and Figures 2018. [Online] Available

18. https://www.cancer.org/content/dam/cancer-org/research/cancer-facts -and-statistics/annual-cancer-facts-and-figures/2018/cancer-facts-andfigures-2018.pdf

19. Last Accessed: 25 th Apr 2020

20. Skin cancer and Facts. What you need to know[Online] Available: https://www.skincancer.org/skin-cancer-information/skin-cancer-facts / Last Accessed: 25 th Jan 2020

21. Skin Cancer[Online] Available: https://www.aad.org/media/stats-skin-cancer Last Accessed: 25 th Apr 2020

22. About melanoma [Online] Available: http://nzmu.co.nz/about-melanoma/ Last Accessed: 25 th Apr 2020

23. Kingma, Diederik \& Ba, Jimmy. (2014). Adam: A Method for Stochastic Optimization. International Conference on Learning Representations.

Published By:

Blue Eyes Intelligence Engineering

\& Sciences Publication

DOI: 10.35940/ijeat.E9514.069520

Journal Website: www.ijeat.org

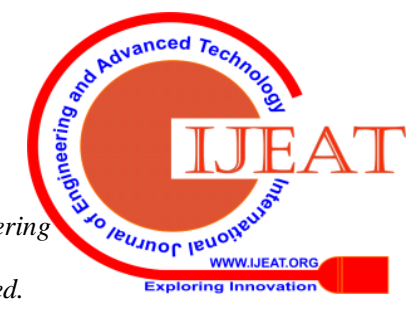


24. Ruder, S. (2016). An overview of gradient descent optimization algorithms. (cite arxiv:1609.04747Comment: Added derivations of AdaMax and Nadam)

25. Tschandl, Philipp, 2018, "The HAM10000 dataset, a large collection of multi-source dermatoscopic images of common pigmented skin lesions" https://doi.org/10.7910/DVN/DBW86T, Harvard Dataverse, $\mathrm{V} 1$,

26. ISIC 2018 [Online] Available: https://challenge2018.isic-archive.com/task3/ Last Accessed:25 th Apr 2020

\section{AUTHORS PROFILE}

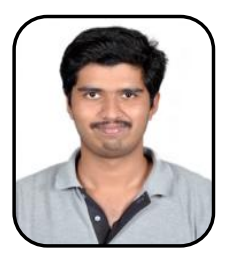

Prasad C Thakar, MTECH, School of Compute Engineering \& Technology, MIT World Peace University, Pune.

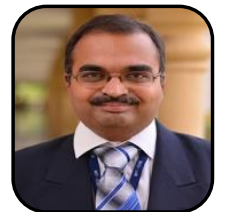

Prof. Dr. Siddhivinayak A Kulkarni, School of Computer Engineering \& Technology, MIT World Peace University, Pune. 